\title{
Identification of target areas for deep brain stimulation in human basal ganglia substructures based on median nerve sensory evoked potential criteria
}

\author{
F Klostermann, J Vesper, G Curio
}

J Neurol Neurosurg Psychiatry 2003;74:1031-1035

See end of article for authors' affiliations

........................

Correspondence to: Dr F Klostermann, Neurophysics Group, Department of Neurology, Universitätsklinikum Benjamin Franklin, Freie Universität Berlin,

Hindenburgdamm 30

12200 Berlin, Germany;

fkloster@zedat.fu-berlin.de

Received

2 December 2002

In revised form

29 January 2003

Accepted 4 March 2003

Objective: In the interventional treatment of movement disorders, the thalamic ventral intermediate nucleus (VIM) and the subthalamic nucleus (STN) are the most relevant electrode targets for deep brain stimulation (DBS). This study tested the value of somatosensory evoked potentials (SEP) for the functional identification of VIM and STN.

Methods: Median nerve SEP were recorded from the final stimulation electrodes targeted at STN and VIM. Throughout the stereotactic procedure SEP were recorded during short electrode stops above STN/VIM and within the presumed target areas. After digital filtering, high and low frequency SEP components were analysed separately to parameterise both the $1000 \mathrm{~Hz}$ SEP burst and low frequency $(<100 \mathrm{~Hz})$ components.

Results: SEP recorded in the VIM target region could unequivocally be distinguished from SEP recorded in STN. The $1000 \mathrm{~Hz}$ burst signal was significantly larger in VIM than in STN without any overlap of amplitude values. In the low frequency band, a primary high amplitude negativity was obtained in VIM, contrasting with a low amplitude positivity in STN. SEP waveshapes in recordings above target positions resembled SEP obtained in STN. When entering VIM, a sharp amplitude increase was observed over a few millimetres only.

Conclusions: Based on SEP criteria, the VIM target but not the STN region can be identified by typical SEP configuration changes, when penetrating the target zone. The approach is independent of the patient's cooperation and vigilance and therefore feasible in general anaesthesia. It provides an easy, reliable, and robust tool for the final assessment of electrode positions at the last instance during electrode implantation when eventual electrode revisions can easily be performed.

O ver the past decade, deep brain stimulation (DBS) has been established as an important therapeutical option for the treatment of various movement disorders, refractory to or complicated by medical treatment..$^{1-3}$ Essential tremor and parkinsonian tremor can be ameliorated or even abolished by DBS of the ventral intermediate nucleus (VIM). ${ }^{4-6}$ In Parkinson's disease (PD) DBS of the subthalamic nucleus (STN) reduces rigor, tremor, and hypokinesia. ${ }^{7-9} \mathrm{~A}$ prerequisite for a beneficial outcome of patients is the accurate identification of a chosen target nucleus so that special emphasis is to be put on procedural aspects of electrode placement. ${ }^{10}{ }^{11}$ Besides brain imaging with ventriculography, CT or MRI, electrophysiological techniques, such as stimulation at and recording from presumed target sites are used intraoperatively as functional targeting devices. ${ }^{12-14}$

Commonly accepted as the the gold standard of recording, high resolution microelectrode derivations are performed to depict target specific neuronal discharge patterns of single units, before implanting the final stimulation electrode, with an ongoing debate on the clinical impact of this technique. ${ }^{15}$ Both the technical equipment and the personal expertise for microrecordings require high standards, which are not easily available.

As, by contrast, the recording of somatosensory evoked potentials (SEP) is electrophysiological routine, we tested intraoperative SEP from the four contact DBS electrode for their anatomical specificity with regard to the ventrolateral thalamus (VIM region) and the subthalamic nucleus (STN region).

\section{METHODS}

In 23 patients with movement disorders (14 Parkinson's disease, six essential tremor, three tremors due to multiple sclerosis; 15 men, 8 women; 34-75 years), undergoing surgery for DBS, SEPs were recorded intraoperatively from the final electrode implant. Ten patients (six with essential tremor, three with multiple sclerosis related tremors, and one with tremor dominant Parkinson's disease) were implanted in VIM, 11 in STN and, early in the series, two Parkinson's disease patients with planned STN surgery in "intermediate" positions (see "Casual observations"). We analysed 29 target SEPs (two SEP per subjects in six bilateral STN implantations, otherwise one SEP per subject). The effect of surgery for patients with Parkinson's disease was rated based on the motor score (part III) of the Unified Parkinson Disease Rating Scale and for tremor patients based on the Essential Tremor Rating Scale. ${ }^{16}{ }^{17}$ All patients gave informed consent under a study protocol approved by the local ethics committee.

The stereotactical procedure was performed from a frontal bur hole. The trajectories to the target points were calculated by image fusion of the preoprative MRI scan and CT scan, the intraoperative ventriculography, and the SchaltenbrandWahren atlas. Targeted regions were accepted or rejected depending on stimulation effects via test micro-electrodes. When-putatively after repeated corrections-the proper target position had been defined, the macro-electrode (with its second but lowest contact) was inserted to this brain site and SEP were recorded during momentary stops $2 \mathrm{~cm}$ and $1 \mathrm{~cm}$ above and within the presumed target position. This means

Abbreviations: VIM, ventral intermediate nucleus; STN, subthalamic nucleus; DBS, deep brain stimulation; SEP, sensory evoked potential 


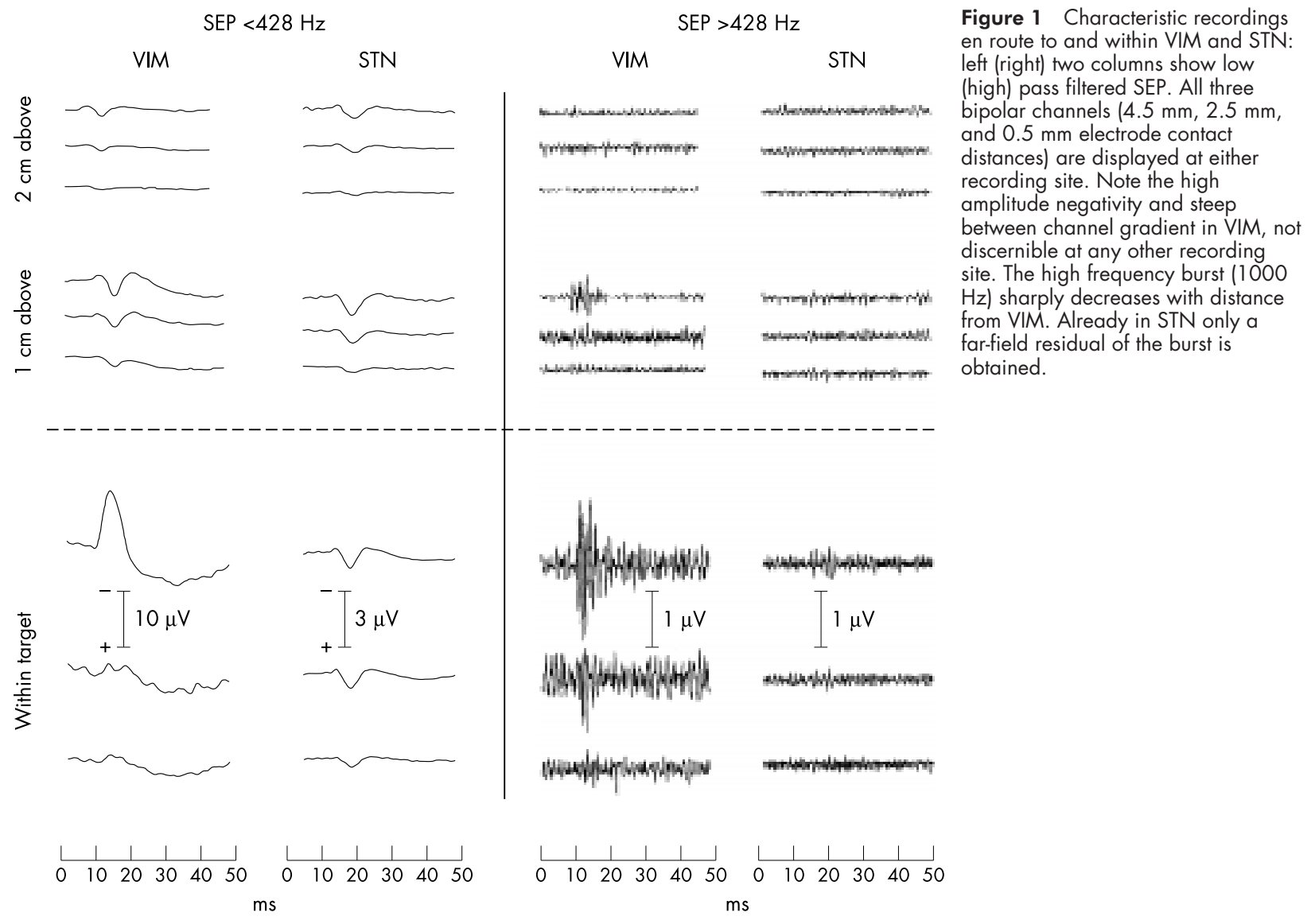

that SEP were collected during the study but not used for targeting. Criteria were developed in a post hoc analysis of the data obtained.

The electrode model (Medtronic 3389) used consists of a shaft with four ring contacts with a width of $1.5 \mathrm{~mm}$, each, spaced at distances of $0.5 \mathrm{~mm}$. The three basal contacts (anodes) were referenced against the cranial one (cathode), resulting in three bipolar derivations over $4.5 \mathrm{~mm}, 2.5 \mathrm{~mm}$, and $0.5 \mathrm{~mm}$. In a fourth channel, the compound action potential was derived mediolaterally at the ipsilateral upper arm proving the constancy of nerval input throughout all recording steps.

The median nerve contralateral to the DBS electrode was stimulated at an intensity of twice motor threshold to guarantee saturated responses. Recordings were performed at the end of surgery under intravenous propofol controlled sedation. Electrical stimuli were applied at $8.1 \mathrm{~Hz}$ (constant current squarewave pulses of $0.1 \mathrm{~ms}$ width) and averaged over 1000 single sweeps. A wide bandpass $(5 \mathrm{~Hz}-1500 \mathrm{~Hz})$ was used for data acquisition (sampling rate $10 \mathrm{kHz}$ ). Offline digital highpass and lowpass filters (corner frequency: $428 \mathrm{~Hz} ; 24$ $\mathrm{dB}$ /octave) were applied to separate high frequency from low frequency responses, which, therefore, could be analysed independently.

Amplitudes of the low frequency subcortical (P16) and cortical (N20) SEP components were determined baseline to peak in the $5 \mathrm{~Hz}-428 \mathrm{~Hz}$ bandpass. The duration and amplitude of the high frequency burst component, superimposed to the primary subcortical component and oscillating around 1000 $\mathrm{Hz}$ for about $5 \mathrm{~ms}-10 \mathrm{~ms}$, was determined in the $428 \mathrm{~Hz}-1500$ $\mathrm{Hz}$ bandpass. $^{18}$ The burst power was determined as a root-mean-square ( $\mathrm{rms}$ ) value over the signal time window and corrected for the noise induced error that was calculated in a burst free time interval from $30 \mathrm{~ms}-45 \mathrm{~ms}$. Amplitude values are given for the first recording channel ( $4.5 \mathrm{~mm}$ grid).
Statistical analyses were performed using two sided paired $t$ tests. Results were considered statistically significant at $\mathrm{p}$ levels $<0.01$.

Electrode positions were determined in a three dimensional system, based on intraoperative radiographs with ventriculography. The anatomical coordinates refer to the position of the second but lowest electrode along the $\mathrm{x}$ axis (laterality), describing the lateral distance from ac-pc midline (line between anterior and posterior commissure), along the y axis (anteriority), defined as the sagittal distance from pc (posterior commissure), and along the $\mathrm{z}$ axis (depth), defined as the vertical distance from ac-pc (negative values indicating below ac-pc/positive values indicating above ac-pc).

\section{RESULTS}

SEP in the VIM region ( $n=10$ of 10 subjects)

SEP recorded from planned VIM targets displayed a specific configuration, distinguishable from SEP waveforms obtained at any other recording site (fig 1 ). In the low frequency band $(<100 \mathrm{~Hz})$ the response is characterised by a single monophasic negative component, sharply rising to an amplitude of 6.9 (5.4) $\mu \mathrm{V}$ (mean (SD)) with a peak latency of 17.3 (2.2) ms. In the high frequency band the $1000 \mathrm{~Hz}$ burst signal exhibited an average rms amplitude of $0.43(0.11) \mu \mathrm{V}$, with signal onset at 13.4 (1.2) $\mathrm{ms}$ and offset at 22.8 (1.6) $\mathrm{ms}$.

The average coordinates for the VIM target were $x=11.6$ (2.2) $\mathrm{mm}, \mathrm{y}=6.6(1.5) \mathrm{mm}$, and $\mathrm{z}=-1.8$ (1) $\mathrm{mm}$. The $\mathrm{z}$ value indicates comparatively low positions. Electrodes targeted to VIM may therefore partially lap into the caudally bordering structure-that is, Zona incerta.

The clinical DBS efficiency was reflected by a decrease of the total tremor score (from 61 (19.1) preoperatively to 24 (18.8) postoperatively; $\left.\mathrm{p}=5.7 \times 10^{-6}\right)$. 


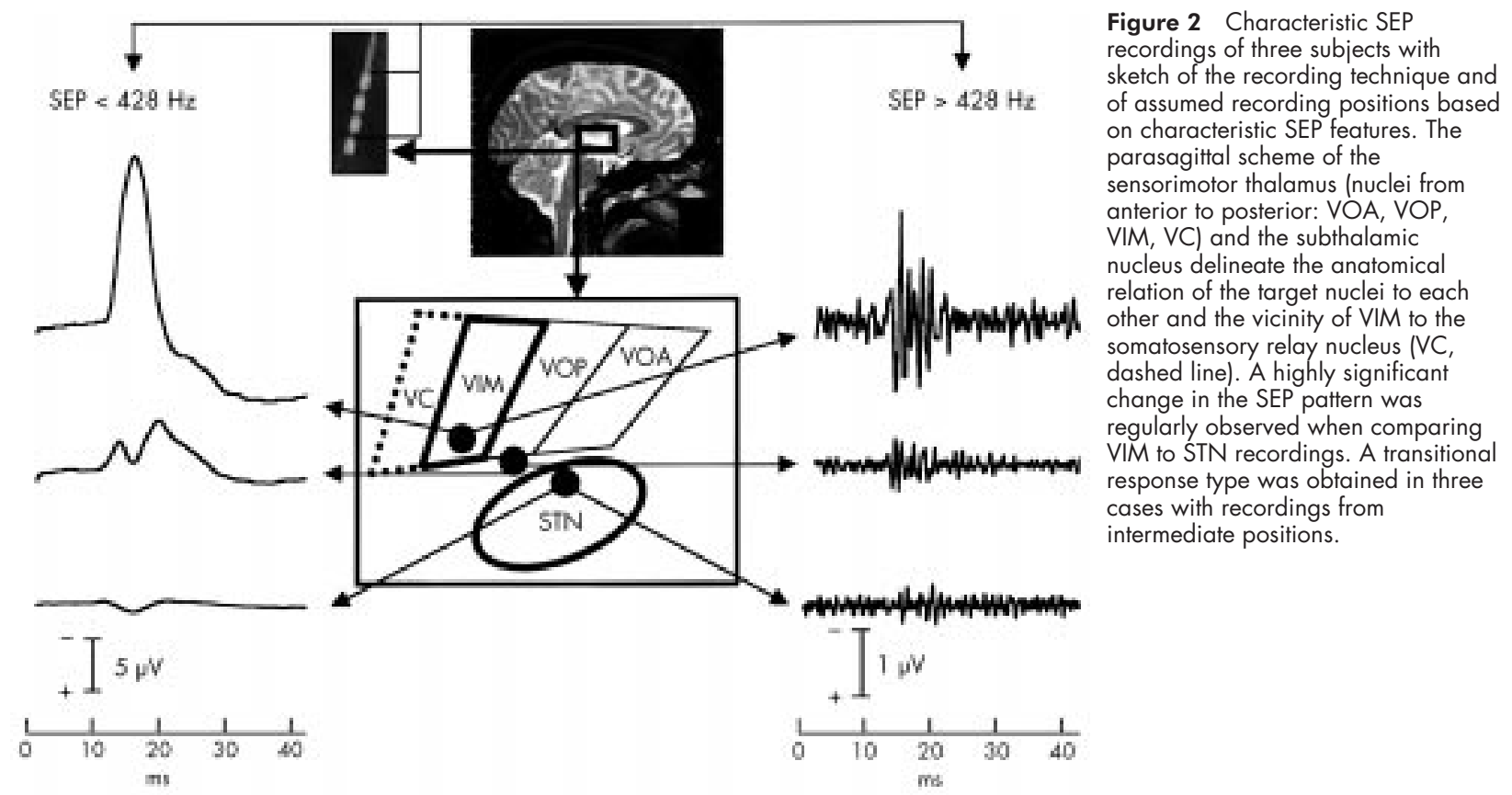

SEP in the STN region ( $n=16$ of 11 subjects)

Low frequency SEP, recorded from planned STN targets, are dominated by a positivity of $1.1(0.4) \mu \mathrm{V}$, peaking at 18.7 (1.4) $\mathrm{ms}$. The $1000 \mathrm{~Hz}$ burst has an amplitude of $0.09(0.02) \mu \mathrm{V}$ with an onset at 14.2 (1.3) $\mathrm{ms}$ and an offset at 22.6 (1.6) $\mathrm{ms}$ (fig l).

The coordinates of the STN targeted electrodes were $\mathrm{x}=11$ (1.4) $\mathrm{mm}, \mathrm{y}=11.1$ (1.7) $\mathrm{mm}$, and $\mathrm{z}=-2.2$ (1.6) $\mathrm{mm}$.

The clinical DBS efficiency was reflected by a reduction on the UPDRS motor scale (37.75 (17.43) preoperatively to 19.1 (16) postoperatively; $\left.\mathrm{p}=4.6 \times 10^{-3}\right)$.

\section{SEP along the trajectory to VIM ( $n=14$ of 7 subjects)} and STN ( $n=32$ of 11 subjects)

SEP recorded $1 \mathrm{~cm}$ and $2 \mathrm{~cm}$ above STN and VIM hardly differed from those obtained in STN. They were characterised by a primary, monophasic positive potential of comparatively low amplitude. When stepping down from $2 \mathrm{~cm}$ to $1 \mathrm{~cm}$ above either target position, an increase in amplitude goes in parallel with a decrease in latency. $1 \mathrm{~cm}$ and $2 \mathrm{~cm}$ above VIM the positive potentials peak at 18.7 (1.9) and 19 (2) ms with amplitudes of 2.4 (1.5) and 1.4 (1) $\mu \mathrm{V}$, respectively. For the STN trajectory the values are: 19.1 (1.6) and 19.8 (1.6) ms with amplitudes of $1.1(0.6)$ and $0.8(0.2) \mu V 4$ - that is, a consistent increase in amplitude cannot be seen towards the target zone. Burst signals are of lower amplitude than in the according target regions, most strikingly in the transition to VIM. The rms amplitudes for high frequency SEP 1 and $2 \mathrm{~cm}$ above VIM are $0.12(0.03)$ and $0.04(0.02) \mu \mathrm{V}$, above STN 0.06 (0.05), and $0.03(0.01) \mu V$, respectively (fig l).

\section{Comparison of VIM SEP and STN SEP}

The SEP amplitudes of low and high frequency responses were significantly different in STN compared with VIM (low frequency responses: $\mathrm{p}=1.2 \times 10^{-3} /$ burst responses: $\left.\mathrm{p}=2.06 \times 10^{-6}\right)$. All low frequency SEP had negative polarity in VIM and positive polarity in STN. High frequency amplitudes did not overlap between targets. Burst responses recorded in VIM are about four times larger than $1 \mathrm{~cm}$ above it, and low frequency components always reverse polarity over this distance. For these low frequency responses, the voltage gradients along the four contact electrode shaft were calculated between electrode contacts 1-4 (used for normalisation), contacts $1-3$, and $1-2$. These gradients were found to differ significantly $(\mathrm{p}<0.01)$ between VIM ( $1: 0.29: 0.03)$ and STN ( $1: 0.78: 0.37)$. Latencies for both components did not differ significantly between the targets.

An interindividually consistent location difference of electrodes was found only for the y coordinate $(4.5 \mathrm{~mm}$; $\left.\mathrm{p}=1.03 \times 10^{-6}\right)$.

\section{Casual observations}

Early in the series in two subjects, exhibiting no or minor clinical gains, SEP did not meet the target criteria described above. Instead, sequences of a negative-positive-negative complex with intermediate amplitudes were recorded (see fig 2 ); this could be correlated to electrode positions between the mean VIM and STN y coordinates in these patients.

In two further patients with planned STN implantations a second surgical intervention was performed for unilateral electrode revisions because of missing clinical stimulation effects. Their SEP configuration resembled a VIM pattern with a monophasic negative component. In both patients the electrodes were shifted to a more anterior position by $3 \mathrm{~mm}$.

Correspondingly, after this correction an STN-like SEP configuration was obtained; clinically, both patients improved.

In one subject an additional recording was performed only $3 \mathrm{~mm}$ above the target position in VIM: this SEP contained a
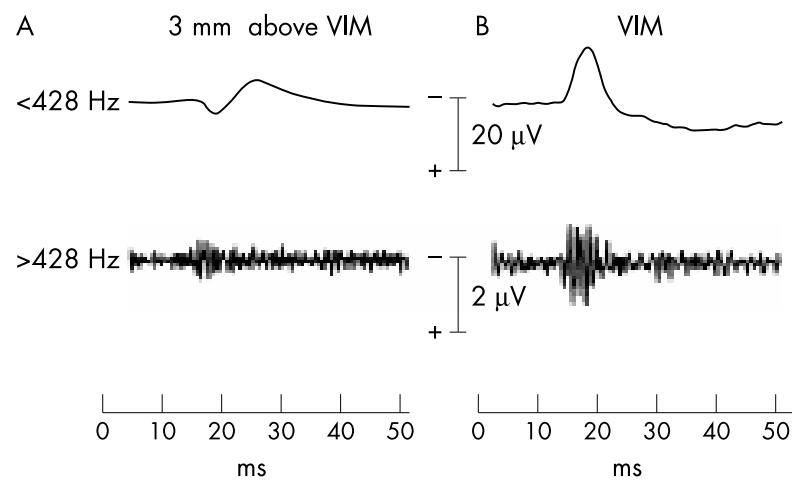

Figure 3 SEP recorded along the trajectory just $3 \mathrm{~mm}$ above VIM (A) and after driving the electrode down into VIM (B). Note the immediate change in amplitude and polarity for the low frequency component and the amplitude increase for the burst response. 
low frequency positivity with the typical waveshape of trajectorial SEP described above. Driving down the electrode $3 \mathrm{~mm}$ into the presumed VIM area, a monophasic negative low frequency response and a high amplitude burst component (15.5 $\mu \mathrm{V}$; burst: $0.51 \mu \mathrm{V}$ ) were obtained (fig 3).

\section{DISCUSSION}

SEP recorded from the definite electrode implant for DBS are reliably indicative for the region targeted for in tremor conditions-that is, VIM/Zona incerta. Parameters of low frequency components and the analysis of recently described local high frequency SEP activity provide useful information for the functional identification of target zones. ${ }^{18}$ This SEP approach is robust against surrounding electromagnetical noise and provides data independent from the patient's vigilance level and cooperation. ${ }^{19}$

The distinct VIM pattern in SEP recordings can be related to the anatomical difference along the y axis. SEP can thus enrich stereotactic surgery by functional data (fig 2 ).

\section{Low frequency SEP $(<100 \mathrm{~Hz})$}

In routine SEP diagnostics, latency and amplitude of low frequency components (for example, N20 in median nerve SEP) are evaluated in a large variety of neurological disorders to assess the integrity of the somatosensory system. They are assumed to reflect mainly postsynaptic potentials. ${ }^{20} 21$

When recording from VIM electrodes, a large monophasic negativity peaking around $17 \mathrm{~ms}$ was obtained, rapidly decreasing just millimetres above VIM as well as more rostrally towards STN. Given the macroscopic nature of the used electrode, we propose this sharply rising component to originate as mixed near-field contributions from the main somatosensory thalamic relay nucleus (VC), dorsally bordering to VIM (see fig 2), and the caudal VIM itself, which also receives short latency somatosensory input. ${ }^{22}$ This assumption is based on the following arguments: (1) the rising SEP amplitude gradient, the closer one records to the neighbouring VC/VIM areas, (2) the phase reversal, occurring on the caudal level of VC/VIM, congruent with earlier semi-microelectrode recordings, ${ }^{22}$ proving short latency somatosensory input as to VIM as to VC, and (3) the steep voltage gradients along the four contact electrode. These findings congruently suggest a near-field origin of the negative wave. Notably, we observed no correlation between SEP recorded in VIM and paraesthesias elicited by DBS.

An intermediate SEP configuration was observed casually along a presumed transition zone between VIM and STN. These SEPs are characterised by a diphasic, primarily negative component.

A monophasic positive SEP component of comparatively small amplitude is typically recorded in STN. This configuration was found at all studied recording sites with the exception of recordings near or in VIM. Thus, the low amplitude profile of SEP recorded along STN trajectories makes the search for SEP peak amplitudes for STN targets a useless enterprise.

\section{High frequency SEP (around $1000 \mathrm{~Hz}$ )}

This burst response of 5-10 oscillatory potentials features fast dynamic characteristics suggesting a generation via synchronised action potentials, eventually from local bursting relay or interneurons and/or the most cranial parts of medial lemniscal fibres. ${ }^{18} 23$

The analysis of this high frequency response has proved a further useful parameter to identify VIM, but only in cases with comparatively low amplitude low frequency SEP. The burst power was severalfold higher in VIM than in surrounding basal ganglia motor structures, including STN, without any overlap. The steep burst increase, moving from any extrathalamic recording site into VIM, hints at a near field generated potential, probably originating from the neighbouring thalamic relay nucleus. ${ }^{24} 25$

\section{Comparison with previous SEP studies}

In contrast with earlier studies on human subcortical SEP, using cephalic or extracephalic skin reference electrodes, intracranial bipolar recordings were analysed in this study. ${ }^{14}{ }^{26-31}$ This technical difference could explain the earlier findings of similar SEP patterns in STN and VIM, distinguishable only from SEP recorded in the somatosensory relay nucleus, whereas with the present bipolar montage a polarity change of primary SEP components could be revealed between VIM and more rostral as well as cranial sites.

Only few data have been published on subcortical high frequency SEP bursts, interpreting them as sequential action potentials, arising in the brain stem, medial lemniscus, and thalamus. ${ }^{26}$ A recent study, using the present bipolar depth recording technique provided support for the hypothesis that the $1000 \mathrm{~Hz}$ burst reflects local rather than propagated activity, ${ }^{25}$ as the narrow bipolar montage (see fig 2) could emphasise near field, high frequency potentials.

\section{Conclusions}

SEP from the final electrode for DBS are a useful tool to provide the therapeutic team with functionally based information on the electrode position. The method is capable of supporting VIM target identification for DBS tremor suppression. Occasionally, it provides a clue for corrections in STN surgery in cases of posterior electrode mislocalisations. SEP are recorded at the last instance when corrections can be achieved without a second surgical approach. The method is appealing for its low demands on equipment and its robustness against electromagnetically noisy surroundings. All SEP evaluation criteria are independent from the patient's vigilance state and cooperation. ${ }^{19}$ SEP from the definite deep brain electrode do not reach the spatial significance of single cell microrecordings, yet they provide a quick and robust information on the momentary electrode placement. Thus, their position in the diagnostic procedure during DBS implantation, in particular the relation between macroscopic SEP and cellular microelectrode recordings, may be worth further study.

\section{Authors' affiliations}

F Klostermann, G Curio, Neurophysics Group, Department of Neurology, Klinikum Benjamin Franklin, Freie Universität, Berlin, Germany

J Vesper, Department of Neurosurgery, Freie Universität, Berlin

Competing interests: none declared.

\section{REFERENCES}

1 Obeso JA, Rodriguez MC, Gorospe A, et al. Surgical treatment of Parkinson's disease. Baillieres Clin Neurol 1997;6:125-45.

2 Benabid AL, Benazzouz A, Limousin P, et al. Dyskinesias and the subthalamic nucleus. Ann Neurol 2000;47 (suppl 1): 189-92.

3 Gross RE, Lozano AM. Advances in neurostimulation for movement disorders. Neurol Res 2000;22:247-58.

4 Hubble JP, Busenbark KL, Wilkinson S, et al. Deep brain stimulation for essential tremor. Neurology 1996;46:1150-3.

5 Ondo W, Almaguer M, Jankovic J, et al. Thalamic deep brain stimulation: comparison between unilateral and bilateral placement. Arch Neurol 2001;58:218-22.

6 Factor SA. Parkinson's disease: motor fluctuations. Current Treatment Options in Neurology 1999;1:21-32.

7 Krack P, Poepping $M$, Weinert $D$, et al. Thalamic, pallidal, or subthalamic surgery for Parkinson's disease? I Neurol 2000;247 (suppl 2): 122-34.

8 Pillon B, Ardouin C, Damier P, et al. Neuropsychological changes between "off" and "on" STN or GPi stimulation in Parkinson's disease. Neurology 2000;55:41 1-18.

9 Vesper J, Klostermann F, Stockhammer F, et al. Results of chronic subthalamic nucleus (STN) stimulation for Parkinson's disease - a one-year follow-up study. Surg Neurol 2002;57:306-13.

10 Starr PA, Vitek JL, Bakay RA. Deep brain stimulation for movement disorders. Neurosurg Clin N Am 1998;9:381-402. 
11 Hariz MI. Pallidotomy microelectrode targeting: neurophysiology-based target refinement. Neurosurgery 2000;47:1472-3,

12 Lemaire JJ, Durif F, Boire JY, et al. Direct stereotactic MRI location in the globus pallidus for chronic stimulation in Parkinson's disease. Acta Neurochir 1999;141:759-65.

13 Yoon MS, Munz M. Placement of deep brain stimulators into the subthalamic nucleus. Stereotact Funct Neurosurg 1999;72:145-9.

14 Hutchison WD, Allan RJ, Opitz H, et al. Neurophysiological identification of the subthalamic nucleus in surgery for Parkinson's disease. Ann Neurol 1998:44:622-8.

15 Hariz MI, Fodstad $\mathrm{H}$. Do microelectrode techniques increase accuracy or decrease risks in pallidotomy and deep brain stimulation? A critical review of the literature. Stereotact Funct Neurosurg 1999;72:157-69.

16 Fahn S, Elton R.1987.. Macmillan health care information. Recent developments in Parkinson's disease. Vol 2. Florham Park, NJ: Macmillan, 1987.

17 Fahn S, Tolosa E, Marin C. Clinical rating scale for tremor. In: Jankovic $\mathrm{J}$, Tolosa E, eds. Parkinson's disease and movement disorders. Baltimore: Urban and Schwarzenberg, 1988:225-34.

18 Klostermann F, Funk T, Vesper J, et al. Spatiotemporal characteristics of human intrathalamic high-frequency $(>400 \mathrm{~Hz})$ SEP components. Neuroreport 1999;10:3627-31

19 Klostermann F, Funk T, Vesper J, et al. Propofol narcosis dissociates human intrathalamic and cortical high-frequency (>400 hz) SEP components. Neuroreport 2000;11:2607-10.

20 Bodis-Wollner I. Sensory evoked potentials: PERG, VEP, and SEP. Curr Opin Neurol Neurosurg 1992;5:716-26.

21 Allison T, Wood CC, McCarthy G, et al. Cortical somatosensory evoked potentials. II. Effects of excision of somatosensory or motor cortex in humans and monkeys. J Neurophysiol 1991;66:64-82.
22 Ohye C, Shibazaki T, Hirai T, et al. Further physiological observations on the ventralis intermedius neurons in the human thalamus. $J$ Neurophysiol 1989;61:488-500.

23 Ramcharan EJ, Gnadt JW, Sherman SM. Burst and tonic firing in thalamic cells of unanesthetized, behaving monkeys. Vis Neurosci 2000; 17:55-62.

24 Gobbele R, Buchner H, Curio G. High-frequency $(600 \mathrm{~Hz})$ SEP activities originating in the subcortical and cortical human somatosensory system. Electroencephalogr Clin Neurophysiol 1998;108:182-9.

25 Klostermann F, Gobbele R, Buchner H, et al. Intrathalamic non-propagating generators of high-frequency $(1000 \mathrm{~Hz})$ somatosensory evoked potential (SEP) bursts recorded subcortically in man. Clin Neurophysiol 2002;113:1001-5.

26 Katayama Y, Tsubokawa T. Somatosensory evoked potentials from the thalamic sensory relay nucleus (VPL) in humans: correlations with short latency somatosensory evoked potentials recorded at the scalp. Electroencephalogr Clin Neurophysiol 1987;68:187-201.

27 Morioka T, Shima F, Kato M, et al. Origin and distribution of thalamic somatosensory evoked potentials in humans. Electroencephalogr Clin Neurophysiol 1989;74:186-93.

28 Yamashiro K, Tasker RR, Iwayama K, et al. Evoked potentials from the human thalamus: correlation with microstimulation and single unit recording. Stereotact Funct Neurosurg 1989;52:127-35.

29 Shima F, Morioka T, Tobimatsu S, et al. Localization of stereotactic targets by microrecordings of thalamic somatosensory evoked potentials. Neurosurgery 1991;28:223-9

30 Taha JM, Favre J, Baumann TK, et al. Functional anatomy of the pallidal base in Parkinson's disease. Neurosurgery 1996;39:1 164-7.

31 Favre J, Taha JM, Baumann T, et al. Computer analysis of the tonic, phasic, and kinesthetic activity of pallidal discharges in Parkinson patients. Surg Neurol 1999;51:665-72.

\section{NEURONLINE}

\section{MS society websites: www.mssociety.org.uk and www.nationalmssociety.org}

$\mathrm{P}$ atients turning up with folders full of printouts from the internet are increasingly common sights in outpatient departments. The response from clinicians often tends towards annoyance and even suspicion. Furthermore, a disease such as multiple sclerosis is linked to much mystery (concerning causes) and controversy (concerning treatments), and as such, the internet abounds with sites making all manner of claims. Physicians therefore need to be able to recommend informative and reliable sites to patients.

The MS Society (UK) provides an easy-to-use and comprehensive web site (www.mssociety.org.uk). Particularly note worthy are downloadable booklets and information sheets. "Newlydiagnosed" patients are sure to find many of these highly useful. Other sections in the web site include links to local support groups, information on working with MS, insurance issues, and pages for carers. The site also gives an overview of research activity in an accessible and balanced way.

Equally impressive is the US equivalent (www.nationalmssociety.org). Again, information is laid out in a user-friendly way with a multitude of links to other web sites pertaining to the disease. Of particular note to the neurologist, however, is the section on clinical study measures, which sets out in

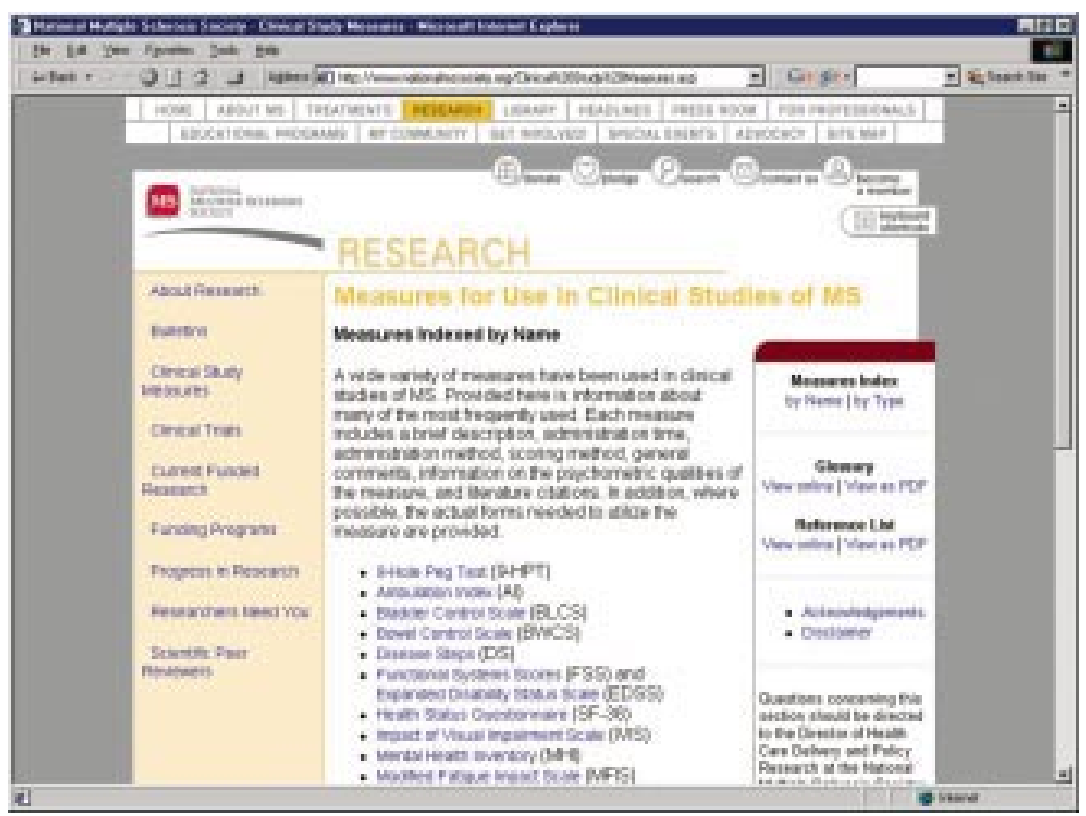

detail, with downloadable forms and user guides, the wide variety of rating scales that have been used in clinical studies of MS, providing a useful resource for all those involved in the management of the disease.

The internet has revolutionised so much of the way we all live our lives, and patients with neurological diseases are increasingly turning to it for information. Control and regulation of information on the internet is a problem, but perhaps if neurologists were to become more involved in writing patient group websites, we could enhance the service we give to patients and prevent some of the more ludicrous medical claims coming to the attention of our patients.

A Wilkins

Department of Neurology, Addenbrooke's Hospital, Hills Road, Cambridge CB2 2QQ, UK' aw255@cam.ac.uk 